\title{
SOME REMARKS ON TSALLIS RELATIVE OPERATOR ENTROPY
}

\author{
SHIGERU FURUICHI AND HAMID REZA MORADI
}

\begin{abstract}
This paper intends to give some new estimates for Tsallis relative operator entropy $T_{v}(A \mid B)=\frac{A \natural_{v} B-A}{v}$. Let $A$ and $B$ be two positive invertible operators with the spectra contained in the interval $J \subset(0, \infty)$. We prove for any $v \in[-1,0) \cup(0,1]$,

$$
\left(\ln _{v} t\right) A+\left(A \natural_{v} B+t A \natural_{v-1} B\right) \leq T_{v}(A \mid B) \leq\left(\ln _{v} s\right) A+s^{v-1}(B-s A)
$$

where $s, t \in J$. Especially, the upper bound for Tsallis relative operator entropy is a non-trivial new result. Meanwhile, some related and new results are also established. In particular, the monotonicity for Tsallis relative operator entropy is improved.

Furthermore, we introduce the exponential type relative operator entropies which are special cases of the perspective and we give inequalities among them and usual relative operator entropies.
\end{abstract}

\section{Introduction And Preliminaries}

Let $\mathcal{B}(\mathcal{H})$ be the algebra of all (bounded linear) operators on a complex Hilbert space $\mathcal{H}$. An operator $A$ on $\mathcal{H}$ is said to be positive (in symbol: $A \geq 0$ ) if $\langle A x, x\rangle \geq 0$ for all $x \in \mathcal{H}$. We write $A>0$ if $A$ is positive and invertible. For self-adjoint operators $A$ and $B$, we write $A \geq B$ if $A-B$ is positive, i.e., $\langle A x, x\rangle \geq\langle B x, x\rangle$ for all $x \in \mathcal{H}$. We call it the usual order. In particular, for some scalars $m$ and $M$, we write $m \leq A \leq M$ if $m\langle x, x\rangle \leq\langle A x, x\rangle \leq M\langle x, x\rangle$

for all $x \in \mathcal{H}$. The spectrum of $A$ is denoted by $S p(A)$. A linear map $\Phi: \mathcal{B}(\mathcal{H}) \rightarrow \mathcal{B}(\mathcal{H})$ is positive if $\Phi(A) \geq 0$ whenever $A \geq 0$. It is said to be unital if $\Phi(I)=I$.

In [5], for $A, B>0$, relative operator entropy was defined by

$$
S(A \mid B)=A^{\frac{1}{2}} \ln \left(A^{-\frac{1}{2}} B A^{-\frac{1}{2}}\right) A^{\frac{1}{2}} .
$$

For $A, B>0$, the Tsallis relative operator entropy is defined as follows (see [13])

$$
T_{v}(A \mid B)=\frac{A \bigsqcup_{v} B-A}{v}=A^{\frac{1}{2}} \ln _{v}\left(A^{-\frac{1}{2}} B A^{-\frac{1}{2}}\right) A^{\frac{1}{2}}, \quad(v \in[-1,0) \cup(0,1]),
$$

2010 Mathematics Subject Classification. Primary 47A63, Secondary 46L05, 47A60.

Key words and phrases. Relative operator entropy; Tsallis relative operator entropy; operator inequality; refined Young inequality. 
where $\ln _{v}(x):=\frac{x^{v}-1}{v}$ is $v$-logarithmic function defined for $x>0$ with $0 \neq v \in \mathbb{R}$ and

$$
A \sharp_{v} B=A^{\frac{1}{2}}\left(A^{-\frac{1}{2}} B A^{-\frac{1}{2}}\right)^{v} A^{\frac{1}{2}}
$$

is the weighted geometric mean for $v \in[0,1]$. Here we use the similar symbol $A \natural_{v} B=$ $A^{\frac{1}{2}}\left(A^{-\frac{1}{2}} B A^{-\frac{1}{2}}\right)^{v} A^{\frac{1}{2}}$ for any $v \in \mathbb{R}$. We remark that

$$
T_{0}(A \mid B)=\lim _{v \rightarrow 0} T_{v}(A \mid B)=S(A \mid B),
$$

since $\lim _{v \rightarrow 0} \ln _{v} t=\ln t$ for $t>0$.

It is known that [9]:

$$
A-A B^{-1} A \leq S(A \mid B) \leq B-A \quad \text { and } \quad A-A B^{-1} A \leq T_{v}(A \mid B) \leq B-A .
$$

Regarding the concavity of $f:[m, M] \rightarrow \mathbb{R}$, we have

$$
\frac{M-t}{M-m} f(m)+\frac{t-m}{M-m} f(M) \leq f(t) \quad \text { for } t \in[m, M] .
$$

Since the function $\ln _{v}(t)=\frac{t^{v}-1}{v}$ on $t>0$ is concave for the case $v \leq 1$, we have

$$
\frac{\ln _{v} m}{M-m}(M A-B)+\frac{\ln _{v} M}{M-m}(B-m A) \leq T_{v}(A \mid B)
$$

whenever $m A \leq B \leq M A$ for some scalars $0<m<M$ and positive invertible operators $A, B$. For the limit of $v \rightarrow 0,(1.2)$ implies

$$
\frac{\ln m}{M-m}(M A-B)+\frac{\ln M}{M-m}(B-m A) \leq S(A \mid B) .
$$

Motivated by the inequality (1.3), Dragomir [2] established some upper and lower bounds for the quantity

$$
S(A \mid B)-\frac{\ln m}{M-m}(M A-B)-\frac{\ln M}{M-m}(B-m A) .
$$

In this note, we present some inequalities for Tsallis relative operator entropy, which are refinements and generalizations of some results obtained by Furuichi et al. [9]. Some related and new inequalities, related to the information monotonicity of Tsallis relative operator entropy, are also established. We also give some alternative bounds for the recent result by Dragomir $[2]$.

\section{Main Results}

At this point, for the reader's convenience, we define the following two abbreviations:

$$
\xi(t)=1+\frac{2^{\frac{t-m}{M-m}}(t-m)(M-t) M^{\frac{t-M}{M-m}}}{(M+m)^{\frac{t+M-2 m}{M-m}}} \quad \text { and } \quad \psi(t)=1+\frac{(t-m)(M-t) M^{\frac{t-M}{M-m}}}{2 m^{\frac{t+M-2 m}{M-m}}}
$$

where $t \in[m, M]$ with $0<m \leq M$. 
Theorem 2.1. Let $A, B \in \mathcal{B}(\mathcal{H})$ be two positive invertible operators satisfying $m A \leq B \leq M A$ for some scalars $0<m<M$. Then

$$
\begin{aligned}
0 & \leq A^{\frac{1}{2}} \ln \xi\left(A^{-\frac{1}{2}} B A^{-\frac{1}{2}}\right) A^{\frac{1}{2}} \\
& \leq S(A \mid B)-\frac{\ln m}{M-m}(M A-B)-\frac{\ln M}{M-m}(B-m A) \\
& \leq A^{\frac{1}{2}} \ln \psi\left(A^{-\frac{1}{2}} B A^{-\frac{1}{2}}\right) A^{\frac{1}{2}}
\end{aligned}
$$

Proof. In the previous paper (see the estimate (2.6) in [7]), the authors stated that if $0<b \leq a$ and $v \in[0,1]$, then

$$
m_{v}\left(\frac{b}{a}\right) a^{1-v} b^{v} \leq(1-v) a+v b \leq M_{v}\left(\frac{b}{a}\right) a^{1-v} b^{v}
$$

where

$$
m_{v}\left(\frac{b}{a}\right)=1+\frac{2^{v} v(1-v)(a-b)^{2}}{a^{1-v}(a+b)^{1+v}} \quad \text { and } \quad M_{v}\left(\frac{b}{a}\right)=1+\frac{v(1-v)(a-b)^{2}}{2 a^{1-v} b^{1+v}}
$$

Assume that $t \in[m, M]$ with $0<m \leq M$. Choosing $a=M, b=m$ and $v=\frac{t-m}{M-m}$, we get

$$
\xi(t) M^{\frac{t-m}{M-m}} m^{\frac{M-t}{M-m}} \leq t \leq \psi(t) M^{\frac{t-m}{M-m}} m^{\frac{M-t}{M-m}}
$$

where $\xi(t)$ and $\psi(t)$ are defined as in (2.1). By taking the logarithm we obtain

$$
\ln \xi(t) \leq \ln t-\frac{\ln m}{M-m}(M-t)-\frac{\ln M}{M-m}(t-m) \leq \ln \psi(t) .
$$

Now, by setting $t=A^{-\frac{1}{2}} B A^{-\frac{1}{2}}$ and then multiplying both sides by $A^{\frac{1}{2}}$ we deduce the desired result.

Remark 2.1. Dragomir in [2, Theorem 2] has proved the following inequalities

$$
\begin{aligned}
0 & \leq K\left(\frac{M}{m}\right)\left(\frac{1}{2} A-\frac{1}{M-m} A^{\frac{1}{2}}\left|A^{-\frac{1}{2}}\left(B-\frac{M+m}{2} A\right) A^{-\frac{1}{2}}\right| A^{\frac{1}{2}}\right) \\
& \leq S(A \mid B)-\frac{\ln m}{M-m}(M A-B)-\frac{\ln M}{M-m}(B-m A) \\
& \leq K\left(\frac{M}{m}\right)\left(\frac{1}{2} A+\frac{1}{M-m} A^{\frac{1}{2}}\left|A^{-\frac{1}{2}}\left(B-\frac{M+m}{2} A\right) A^{-\frac{1}{2}}\right| A^{\frac{1}{2}}\right) .
\end{aligned}
$$

To compare Theorem 2.1 and Dragomir's result, it is sufficient to compare the following inequalities for $0<x \leq 1$ :

$$
m_{v}(x) x^{v} \leq(1-v)+v x \leq M_{v}(x) x^{v}
$$

where

$$
m_{v}(x)=1+\frac{2^{v} v(1-v)(x-1)^{2}}{(x+1)^{v+1}} \quad \text { and } \quad M_{v}(x)=1+\frac{v(1-v)(x-1)^{2}}{2 x^{v+1}}
$$


and

$$
K^{r}(x) x^{v} \leq(1-v)+v x \leq K^{R}(x) x^{v},
$$

where $v \in[0,1], r=\min \{v, 1-v\}, R=\max \{v, 1-v\}$ and $K(x)=\frac{(x+1)^{2}}{4 x}$. As we mentioned in [7, Proposition 3.1], there is no ordering between $K^{r}(x)$ and $m_{v}(x)$ (and also between $K^{r}(x)$ and $\left.M_{v}(x)\right)$. Therefore we conclude that Theorem 2.1 is not a trivial result.

It seems worthwhile to point out the following remark.

Remark 2.2. Let $v \in(0,1]$. As we mentioned in [12, Remark 1], for $x \geq 1$ we have

$$
0 \leq 1-\frac{1}{x} \leq\left(\frac{x+1}{2}\right)^{v-1}(x-1) \leq \frac{x^{v}-1}{v} \leq\left(\frac{x^{v-1}+1}{2}\right)(x-1) \leq x-1 .
$$

It follows from (2.2) that

$$
1-\frac{1}{x} \leq \frac{1}{M_{v}(x)}\left(\frac{1-v}{v}+x\right)-\frac{1}{v} \leq \frac{x^{v}-1}{v} \leq \frac{1}{m_{v}(x)}\left(\frac{1-v}{v}+x\right)-\frac{1}{v} \leq x-1<0,
$$

where $0<x \leq 1$ and $0<v \leq 1$.

Actually, the first inequality in (2.4) is equivalent to the inequality

$$
v(x-1)^{2} g(v, x) \geq 0, \quad g(v, x) \equiv 2 x^{v+1}-(1-v)\{(1+v) x-v\}
$$

so that we have only to prove $g(v, x) \geq 0$ for $0<v \leq 1$ and $0<x \leq 1$.

Since $\frac{d g(v, x)}{d x}=(1+v)\left(2 x^{v}-1+v\right)$ and $\frac{d^{2} g(v, x)}{d x^{2}}=2 v(1+v) x^{v-1} \geq 0$, we have minimum value of the function $g(v, x)$ when $x=\left(\frac{1-v}{2}\right)^{1 / v}$ and it is calculated as

$$
g\left(v,\left(\frac{1-v}{2}\right)^{1 / v}\right)=v(1-v)\left\{1-\left(\frac{1-v}{2}\right)^{1 / v}\right\} \geq 0 .
$$

The second and third inequalities in (2.4) are straightforward from (2.2). The forth inequality in (2.4) is equivalent to the inequality for $x>0$ and $0<v \leq 1$,

$$
\frac{\left(m_{v}(x)-1\right)}{m_{v}(x)}\left(\frac{(1-v)+v x}{v}\right) \geq 0
$$

by simple calculations.

Remark 2.3. We know that (see [11, Remark 2.11]) if $x>0$ and $v \notin[0,1]$, then

$$
(1-v)+v x \leq x^{v}
$$

The function $f_{v}(t)=\frac{v(1-v)(t-1)}{t^{v+1}}$ is convex for all $t>0$ and $v \in[-1,0]$. This follows from the following fact

$$
f_{v}^{\prime \prime}(t)=\frac{v(v+1)(1-v)(v(x-1)-2)}{x^{v+3}} \geq 0 .
$$


Using the Hermite-Hadamard inequality, one can get

$$
f_{v}\left(\frac{1+x}{2}\right) \leq \frac{1}{1-x} \int_{x}^{1} f_{v}(t) d t \leq \frac{f_{v}(1)+f_{v}(x)}{2}
$$

for each $0<x \leq 1$ and $v \in[-1,0]$. The above inequality entails that

$$
M_{v}(x) \leq \frac{(1-v)+v x}{x^{v}} \leq m_{v}(x)
$$

where $M_{v}(x)$ and $m_{v}(x)$ are defined as in (2.3).

Notice that for $0<x \leq 1$ and $v \in[-1,0]$, the functions $m_{v}(x)$ and $M_{v}(x)$ are increasing and $0<M_{v}(x) \leq m_{v}(x) \leq 1$ (similar to that of [7, Proposition 2.4], so we omit details). Thus, inequality (2.6) provides a refinement and a reverse for the inequality (2.5).

Theorem 2.2. Let $A, B \in \mathcal{B}(\mathcal{H})$ be two positive invertible operators such that $\operatorname{Sp}(A), S p(B) \subseteq$ $J \subset(0, \infty)$. Then

$$
\left(\ln _{v} t\right) A+\left(A \natural_{v} B-t A \natural_{v-1} B\right) \leq T_{v}(A \mid B) \leq\left(\ln _{v} s\right) A+s^{v-1}(B-s A),
$$

for any $s, t \in J$ and $v \in[-1,0) \cup(0,1]$.

Proof. The differentiable function $f$ is concave on $J$ if, for any $s \in J$, the tangent line through $(s, f(s))$ is above the graph of $f$. That is

$$
f(t) \leq f(s)+f^{\prime}(s)(t-s) .
$$

Of course, the function $f(x)=\ln _{v} x \quad(v \in[-1,0) \cup(0,1])$ is differentiable and concave so

$$
\ln _{v} t \leq \ln _{v} s+s^{v-1}(t-s)
$$

for any $s \in J$.

Applying functional calculus for the positive operator $A^{-\frac{1}{2}} B A^{-\frac{1}{2}}$, we get

$$
\ln _{v}\left(A^{-\frac{1}{2}} B A^{-\frac{1}{2}}\right) \leq\left(\ln _{v} s\right) I+s^{v-1}\left(A^{-\frac{1}{2}} B A^{-\frac{1}{2}}-s I\right)
$$

for any $s \in J$. By multiplying both sides by $A^{\frac{1}{2}}$ we deduce the second inequality.

On the other hand, from inequality (2.7), we get

$$
\left(\ln _{v} t\right) I \leq \ln _{v}\left(A^{-\frac{1}{2}} B A^{-\frac{1}{2}}\right)+t\left(A^{-\frac{1}{2}} B A^{-\frac{1}{2}}\right)^{v-1}-\left(A^{-\frac{1}{2}} B A^{-\frac{1}{2}}\right)^{v} .
$$

Multiplying $A^{\frac{1}{2}}$ from both sides, we have the first inequality.

Remark 2.4. In [9, Theorem 3.6], Furuichi et.al. obtained the following inequality:

$$
A \sharp_{v} B-\frac{1}{\alpha} A \bigsqcup_{v-1} B+\left(\ln _{v} \frac{1}{\alpha}\right) A \leq T_{v}(A \mid B) \leq \frac{1}{\alpha} B-A-\left(\ln _{v} \frac{1}{\alpha}\right) A \sharp_{v} B,
$$

for $\alpha>0$ and $0<v \leq 1$. We see the first inequality of (2.8) is just same to one in Theorem 2.2 with $t=\frac{1}{\alpha}$. However, its proof is an entirely different proof to [9, Theorem 3.6]. 
The second inequalities of (2.8) and in Theorem 2.2 with $s=\frac{1}{\alpha}$ are different. In order to compare these, we have to compare $g_{v}(s, t):=\left(\ln _{v} s\right)+s^{v-1} t-s^{v}$ and $h_{v}(s, t):=s t-1-\left(\ln _{v} s\right) t^{v}$ for $v \in[-1,0) \cup(0,1]$ and $s, t>0$. However there is no ordering between them. Actually, we have $f_{0.5}(0.1,1) \simeq 1.01096$ and $f_{0.5}(0.1,0.1) \simeq-0.81$, where $f_{v}(s, t):=g_{v}(s, t)-h_{v}(s, t)$. In addition our Theorem 2.2 holds for $v \in[-1,0) \cup(0,1]$, while the inequalities (2.8) given in $[9$, Theorem 3.6] were shown for $v \in(0,1]$. Therefore our obtained upper bound for $T_{v}(A \mid B)$ in Theorem 2.2 is not trivial result.

For the limit of $v \rightarrow 0$ in Theorem 2.2 we have

$$
(\log t) A+\left(A-t A B^{-1} A\right) \leq S(A \mid B) \leq(\log s) A+s^{-1} B-A .
$$

In addition, if we take $t=\frac{1}{\alpha}$ and $s=\alpha$ in the above, then we obtain the known inequalities

$$
(1-\log \alpha) A-\frac{1}{\alpha} A B^{-1} A \leq S(A \mid B) \leq(\log \alpha-1) A+\frac{1}{\alpha} .
$$

Furthermore, if we put $\alpha=1$ above, then we recover

$$
A-A B^{-1} A \leq S(A \mid B) \leq B-A .
$$

See [9] for these known inequalities, for example.

Extending a work of Fujii [6] for relative operator entropy, Furuichi et al. [9, Proposition 2.3] obtained:

$$
\Phi\left(T_{v}(A \mid B)\right) \leq T_{v}(\Phi(A) \mid \Phi(B))
$$

where $\Phi: \mathcal{B}(\mathcal{H}) \rightarrow \mathcal{B}(\mathcal{H})$ is a unital positive linear map. In the following, we try to improve inequality (2.9), which is often called the monotonicity for Tsallis relative operator entropy.

Theorem 2.3. Let $A, B \in \mathcal{B}(\mathcal{H})$ be two positive invertible operators and let $\Phi: \mathcal{B}(\mathcal{H}) \rightarrow \mathcal{B}(\mathcal{H})$ be a unital positive linear map. Then for any $v \in[0,1]$

$$
\begin{aligned}
\Phi\left(T_{v}(A \mid B)\right) & \leq \int_{0}^{1} \frac{\Phi\left(\left(A \sharp_{v} B\right) \sharp_{\mu} A\right) \sharp_{v} \Phi\left(\left(A \sharp_{v} B\right) \sharp_{\mu} B\right)-\Phi(A)}{v} d \mu \\
& \leq T_{v}(\Phi(A) \mid \Phi(B)) .
\end{aligned}
$$

Proof. First, we need the following inequality from [11, Theorem 3.1], which connects both sides of the Ando's inequality [1]:

$$
\Phi\left(A \sharp_{v} B\right) \leq \Phi\left(\left(A \sharp_{v} B\right) \sharp_{\mu} A\right) \sharp_{v} \Phi\left(\left(A \sharp_{v} B\right) \sharp_{\mu} B\right) \leq \Phi(A) \sharp_{v} \Phi(B)
$$

for $v, \mu \in[0,1]$. Integrating the inequality (2.10) over $\mu \in[0,1]$, we obtain

$$
\Phi\left(A \sharp_{v} B\right) \leq \int_{0}^{1} \Phi\left(\left(A \sharp_{v} B\right) \sharp_{\mu} A\right) \sharp_{v} \Phi\left(\left(A \sharp_{v} B\right) \sharp_{\mu} B\right) d \mu \leq \Phi(A) \sharp_{v} \Phi(B) .
$$


By virtue of (2.11), we have

$$
\begin{aligned}
\frac{\Phi\left(A \sharp_{v} B\right)-\Phi(A)}{v} & \leq \frac{\int_{0}^{1} \Phi\left(\left(A \sharp_{v} B\right) \sharp_{\mu} A\right) \sharp_{v} \Phi\left(\left(A \sharp_{v} B\right) \sharp_{\mu} B\right) d \mu-\Phi(A)}{v} \\
& \leq \frac{\Phi(A) \sharp_{v} \Phi(B)-\Phi(A)}{v} .
\end{aligned}
$$

It follows from the linearity of $\Phi$ that $\Phi\left(\frac{A \sharp_{v} B-A}{v}\right)=\frac{\Phi\left(A \sharp_{v} B\right)-\Phi(A)}{v}$. This completes the proof.

We close this section by giving a complementary inequality of (2.9).

Proposition 2.1. Let $A, B \in \mathcal{B}(\mathcal{H})$ be two positive invertible operators such that $S p(A), S p(B) \subseteq$ $J$ and let $\Phi: \mathcal{B}(\mathcal{H}) \rightarrow \mathcal{B}(\mathcal{H})$ be a unital positive linear map. Then

$$
\begin{aligned}
T_{v}(\Phi(A) \mid \Phi(B)) \leq & \Phi\left(T_{v}(A \mid B)\right)+\left(\ln _{v} s-\ln _{v} t\right) \Phi(A) \\
& +\Phi\left(t A \natural_{v-1} B-A \sharp_{v} B\right)+s^{v-1} \Phi(B-s A)
\end{aligned}
$$

for any $s, t \in J$ and $v \in[0,1]$.

Proof. It follows from the first inequality in Theorem 2.2 that

$$
\left(\ln _{v} t\right) \Phi(A)+\Phi\left(A \sharp_{v} B-t A \natural_{v-1} B\right) \leq \Phi\left(T_{v}(A \mid B)\right) .
$$

This implies

$$
0 \leq \Phi\left(T_{v}(A \mid B)\right)-\left(\ln _{v} t\right) \Phi(A)-\Phi\left(A \sharp_{v} B-t A \natural_{v-1} B\right) .
$$

On the other hand, the second inequality in Theorem 2.2 implies

$$
T_{v}(\Phi(A) \mid \Phi(B)) \leq\left(\ln _{v} s\right) \Phi(A)+s^{v-1} \Phi(B-s A) .
$$

Adding (2.13) to (2.14), we infer the desired inequality.

Remark 2.5. For the limit of $v \rightarrow 0$ in Proposition 2.1, we obtain the inequality:

$$
S(\Phi(A) \mid \Phi(B)) \leq \Phi(S(A \mid B))+\left(\log \frac{s}{t}-2\right) \Phi(A)+\Phi\left(t A B^{-1} A-s^{-1} B\right) .
$$

\section{ANOTHER LOOK AT TSALLIS RELATIVE OPERATOR ENTROPY}

Entropies are usually defined by the use of logarithmic functions. They can be redefined by the use of exponential functions in artificially formal. We study relative operator entropies defined by exponential functions and give some operator inequalities for them. We here use $v$-exponential function defined by $\exp _{v}(x):=(1+v x)^{1 / v}$ for $x>0$ and $v \in[-1,0) \cup(0,1]$. The function $\exp _{v}$ is the inverse of $\ln _{v}$, and we have $\lim _{v \rightarrow 0} \exp _{v}(x)=\exp (x)$.

It is known that Shannon entropy is defined by logarithmic function as $H(\mathbf{p})=-\sum_{j=1}^{n} p_{j} \log p_{j}$ for probability distribution $\mathbf{p}=\left(p_{1}, \cdots, p_{n}\right)$, where $p_{j} \geq 0$ and $\sum_{j=1}^{n} p_{j}=1$. Putting 
$p_{j}=e^{-s_{j}}$, we can rewrite $H(\mathbf{p})$ as $H^{\exp }(\mathbf{s})=\sum_{j=1}^{n} s_{j} e^{-s_{j}}$, with $s_{j} \geq 0$. For Tsallis entropy $T_{v}(\mathbf{p})=\sum_{j=1}^{n} p_{j} \ln _{v} \frac{1}{p_{j}}$ can be rewritten as $T_{v}^{\exp }(\mathbf{s})=\sum_{j=1}^{n} s_{j} \exp _{-v}\left(-s_{j}\right)$ with $s_{j} \geq 0$, by putting $\frac{1}{p_{j}}=\exp _{v}\left(s_{j}\right)$. Similar modifications happen for the relative entropy $D(\mathbf{p} \mid \mathbf{q})=$ $\sum_{j=1}^{n} p_{j}\left(\log p_{j}-\log q_{j}\right)$ and the Tsallis relative entropy $T_{v}(\mathbf{p} \mid \mathbf{q})=\sum_{j=1}^{n} p_{j}^{1-r}\left(\ln _{v} p_{j}-\ln _{v} q_{j}\right)$, for probability distribution $\mathbf{q}=\left(q_{1}, \cdots, q_{n}\right)$, where $q_{j} \geq 0$ and $\sum_{j=1}^{n} q_{j}=1$. By putting actually $p_{j}=e^{-s_{j}}$ and $q_{j}=e^{-t_{j}}$, we can rewrite the relative entropy as $D^{\exp }(\mathbf{s} \mid \mathbf{t})=\sum_{j=1}^{n}\left(t_{j}-s_{j}\right) e^{-s_{j}}$. Putting by $\frac{1}{p_{j}}=\exp _{v}\left(s_{j}\right)$ and $\frac{1}{q_{j}}=\exp _{v}\left(t_{j}\right)$, we can rewrite the Tsallis relative entropy as $D_{v}^{e x p}(\mathbf{s} \mid \mathbf{t})=\sum_{j=1}^{n}\left\{\left(\frac{\exp _{-v}\left(-t_{j}\right)}{\exp _{-v}\left(-s_{j}\right)}\right)^{v} t_{j}-s_{j}\right\} \exp _{-v}\left(-s_{j}\right)$. We note that

$$
\lim _{v \rightarrow 0} T_{v}^{\exp }(\mathbf{s})=H^{\exp }(\mathbf{s}) \quad \text { and } \quad \lim _{v \rightarrow 0} D_{r}^{\exp }(\mathbf{s} \mid \mathbf{t})=D^{\exp }(\mathbf{s} \mid \mathbf{t})
$$

as known $\lim _{v \rightarrow 0} T_{v}(\mathbf{p})=H(\mathbf{p})$ and $\lim _{v \rightarrow 0} T_{v}(\mathbf{p} \mid \mathbf{q})=D(\mathbf{p} \mid \mathbf{q})$.

As we have seen some entropies can be written by $\exp (x)$ and $\exp _{v}(x)$ without $\ln (x)$ and $\ln _{r}(x)$. However we give nothing for information theoretical insights for our quantities. In this note, we just give mathematical inequalities for the relative operator entropies redefined by $\exp (x)$ and $\exp _{v}(x)$.

We give scalar inequalities for the function $\exp _{v}(t)$ below.

Lemma 3.1. Let $t>0$, then

$$
\begin{gathered}
t \exp _{v / 2}(t)^{\frac{1-v}{2}} \leq \exp _{v}(t)-1 \leq \frac{t}{2}\left(1+\exp _{v}(t)^{1-v}\right), \quad(-1 \leq v<0), \\
\frac{t}{2}\left(1+\exp _{v}(t)^{1-v}\right) \leq \exp _{v}(t)-1 \leq t \exp _{v / 2}(t)^{\frac{1-v}{2}}, \quad(0<v \leq 1) .
\end{gathered}
$$

Proof. Consider the function $f_{v}(t)=(1+v t)^{\frac{1-v}{v}}$ where $t>0$ and $v \in[-1,0) \cup(0,1]$. We have that

$$
\left\{\begin{array}{rr}
f^{\prime \prime}{ }_{v}(t) \geq 0 & (-1 \leq v<0) \\
f^{\prime \prime}{ }_{v}(t) \leq 0 & (0<v \leq 1)
\end{array} .\right.
$$

Now using the well-known Hermite-Hadamard inequality for convex (concave) function $f_{v}(t)$, we infer the desired result.

Utilizing the definition of the $v$-exponential function $\exp _{v}(t)$ defined for $t>0$ and $v \in$ $[-1,0) \cup(0,1]$, we can define

$$
\mathcal{E}_{v}(A \mid B):=A^{\frac{1}{2}} \exp _{v}\left(A^{-\frac{1}{2}} B A^{-\frac{1}{2}}\right) A^{\frac{1}{2}},
$$

where $A, B \in \mathcal{B}(\mathcal{H})$ are two positive invertible operators. It is easy to see that $\lim _{v \rightarrow 0} \exp _{v}(t)=$ $\exp (t)$. So we have

$$
\lim _{v \rightarrow 0} \mathcal{E}_{v}(A \mid B)=A^{\frac{1}{2}} \exp \left(A^{-\frac{1}{2}} B A^{-\frac{1}{2}}\right) A^{\frac{1}{2}}=: \mathcal{E}(A \mid B)
$$

It is notable $\mathcal{E}(A \mid B)$ and $\mathcal{E}_{v}(A \mid B)$ are special cases for the perspective [3, 4]. Lemma 3.1 implies the following operator inequalities. 
Theorem 3.1. Let $A, B \in \mathcal{B}(\mathcal{H})$ be positive invertible operators. If $-1 \leq v<0$, then

$$
B A^{-1} \mathcal{E}_{v / 2}(A \mid B)^{\frac{1-v}{2}}+A \leq \mathcal{E}_{v}(A \mid B) \leq A+\frac{1}{2} B+\frac{1}{2} B A^{-1} \mathcal{E}_{v}(A \mid B)^{1-v} .
$$

If $0<v \leq 1$, then

$$
A+\frac{1}{2} B+\frac{1}{2} B A^{-1} \mathcal{E}_{v}(A \mid B)^{1-v} \leq \mathcal{E}_{v}(A \mid B) \leq B A^{-1} \mathcal{E}_{v / 2}(A \mid B)^{\frac{1-v}{2}}+A .
$$

We also have the following relations among four operators.

Theorem 3.2. Let $A, B \in \mathcal{B}(\mathcal{H})$ be positive invertible operators, then

$$
\begin{gathered}
S(A \mid B) \leq T_{v}(A \mid B) \leq \mathcal{E}_{v}(A \mid B) \leq \mathcal{E}(A \mid B), \quad(0<v \leq 1) \\
\mathcal{E}(A \mid B) \leq \mathcal{E}_{v}(A \mid B) \leq T_{v}(A \mid B) \leq S(A \mid B), \quad\left(-1 \leq v<0, \quad v \neq-\frac{1}{2}\right)
\end{gathered}
$$

where $S(A \mid B)$ and $T_{v}(A \mid B)$ are relative operator entropy [14] and Tsallis relative operator entropy [16], respectively.

Proof. The first inequality in (3.2) and the last inequality in (3.3) follows directly from [15, Proposition 3.1]. We know that for each $t>0$,

$$
\begin{gathered}
\exp _{v}(t) \leq \exp (t), \quad(0<v \leq 1) \\
\exp (t) \leq \exp _{v}(t), \quad\left(-1 \leq v<0, v \neq-\frac{1}{2}\right),
\end{gathered}
$$

these gives the last and the first inequality in (2.1) and (3.3), respectively. On the other hand for each $t>0$,

$$
\begin{gathered}
\ln _{v}(t) \leq \exp _{v}(t), \quad(0<v \leq 1) \\
\exp _{v}(t) \leq \ln _{v}(t), \quad\left(-1 \leq v<0, \quad v \neq-\frac{1}{2}\right)
\end{gathered}
$$

Theorem 3.3. Let $B \leq C$. If $\frac{m-1}{v} A \leq B \leq \frac{M-1}{v} A$ with $0<m<M$, then

$$
\mathcal{E}_{v}(A \mid B) \leq K(m, M, v) \mathcal{E}_{v}(A \mid C), \quad(v \in[-1,0) \cup(0,1])
$$

where

$$
K(m, M, v) \equiv \frac{\left(m M^{v}-M m^{v}\right)}{(v-1)(M-m)}\left(\frac{(v-1)\left(M^{v}-m^{v}\right)}{v\left(m M^{v}-M m^{v}\right)}\right)^{v}>0 .
$$


Proof. According to [17, Theorem 1.5],

$$
\left\langle\left(I+v A^{-\frac{1}{2}} B A^{-\frac{1}{2}}\right)^{\frac{1}{v}} x, x\right\rangle \leq K(m, M, v)\left\langle\left(I+v A^{-\frac{1}{2}} B A^{-\frac{1}{2}}\right) x, x\right\rangle^{\frac{1}{v}},
$$

holds for any unit vector $x \in \mathcal{H}$ and $v \in[-1,0) \cup(0,1]$.

So we have

$$
\begin{aligned}
\frac{1}{K(m, M, v)}\left\langle\left(I+v A^{-\frac{1}{2}} B A^{-\frac{1}{2}}\right)^{\frac{1}{v}} x, x\right\rangle & \leq\left\langle\left(I+v A^{-\frac{1}{2}} B A^{-\frac{1}{2}}\right) x, x\right\rangle^{\frac{1}{v}} \\
& \leq\left\langle\left(I+v A^{-\frac{1}{2}} C A^{-\frac{1}{2}}\right) x, x\right\rangle^{\frac{1}{v}} \\
& \leq\left\langle\left(I+v A^{-\frac{1}{2}} C A^{-\frac{1}{2}}\right)^{\frac{1}{v}} x, x\right\rangle .
\end{aligned}
$$

Whence

$$
\left(I+v A^{-\frac{1}{2}} B A^{-\frac{1}{2}}\right)^{\frac{1}{v}} \leq K(m, M, v)\left(I+v A^{-\frac{1}{2}} C A^{-\frac{1}{2}}\right)^{\frac{1}{v}} .
$$

Multiplying both sides by $A^{\frac{1}{2}}$ we deduce the desired inequality (3.4).

\section{ACKNOWLEDGMENT}

The author (S.F.) was partially supported by JSPS KAKENHI Grant Number 16K05257.

\section{REFERENCES}

[1] T. Ando, X. Zhan, Norm inequalities related to operator monotone functions, Math Ann., 315 (1999), $771-780$.

[2] S.S. Dragomir, Some inequalities for relative operator entropy, Preprint RGMIA Res. Rep. Col 1. 18, Art. 145 (2015) [http://rgmia.org/papers/v18/v18a145.pdf].

[3] A. Ebadian, I. Nikoufar, M. E. Gordji, Perspectives of matrix convex functions, Proc. Natl. Acad. Sci. USA., 108 (2011), 7313-7314.

[4] E. Effros, F. Hansen, Non-commutative perspectives, Ann. Funct. Anal. 5(2014), 74-79.

[5] J.I. Fujii, E. Kamei, Relative operator entropy in noncommutative information theory, Math. Japon., 34 (1989), 341-348.

[6] J.I. Fujii, Operator means and the relative operator entropy, Oper. Theory Adv. Appl., 59 (1992), 161-172.

[7] S. Furuichi, H.R. Moradi, Some refinements of classical inequalities, Rocky Mountain J. Math., 48(7) (2018), 2289-2309.

[8] S. Furuichi, H.R. Moradi, On further refinements for Young inequalities, Open Math., 16 (2018), 1478-1482.

[9] S. Furuichi, K. Yanagi, K. Kuriyama, A note on operator inequalities of Tsallis relative operator entropy, Linear Algebra Appl., 407 (2005), 19-31.

[10] H.R. Moradi, S. Furuichi, A complementary inequality to the information monotonicityfor Tsallis relative operator entropy, Linear Multilinear Algebra., https://doi.org/10.1080/03081087.2018.1549532

[11] H.R. Moradi, S. Furuichi, M. Sababheh, Some inequalities for interpolational operator means, arXiv:1808.08342. 
[12] H.R. Moradi, S. Furuichi, N. Minculete, Estimates for Tsallis relative operator entropy, Math. Inequal. Appl., 20(4) (2017), 1079-1088.

[13] K. Yanagi, K. Kuriyama, S. Furuichi, Generalized Shannon inequality based on Tsallis relative operator entropy, Linear Algebra Appl., 394 (2005), 109-118.

[14] J.I. Fujii, E. Kamei, Relative operator entropy in noncommutative information theory, Math. Japon., 34 (1989), 341-348.

[15] S. Furuichi, K. Yanagi, K. Kuriyama, A note on operator inequalities of Tsallis relative operator entropy, Linear Algebra Appl., 407 (2005), 19-31.

[16] K. Yanagi, K. Kuriyama, S. Furuichi, Generalized Shannon inequalities based on Tsallis relative operator entropy, Linear Algebra Appl., 394 (2005), 109-118.

[17] T. Furuta, Operator inequalities associated with Hölder-McCarthy and Kantorovich inequalities, J. Inequal. Appl., 2 (1998), 137-148.

(S. Furuichi) Department of Information Science, College of Humanities and Sciences, Nihon University, 3-25-40, Sakurajyousui, Setagaya-ku, Tokyo, 156-8550, Japan.

E-mail address: furuichi@chs.nihon-u.ac.jp

(H. R. Moradi) Department of Mathematics, Payame Noor University (PNU), P.O.Box, 19395-4697, Tehran, Iran.

E-mail address: hrmoradi@mshdiau.ac.ir 